\title{
Effects of S-adenosyl-L-Methionine Combined with Ursodesoxycholic Acid on Serum Endotoxin, MMP-9 and IL-18 in Neonates with Cholestasis
}

\author{
Lingmei Zeng ${ }^{1}$, Mao $\mathrm{Li}^{1}$, Yuting Cen ${ }^{1}$ and Zuanyu Wang ${ }^{2}$ \\ ${ }^{1}$ Department of Neonatology, Luzhou People's Hospital, Luzhou, Sichuan Province, China \\ ${ }^{2}$ Department of Hepatobiliary Pancreatic and Splenic Surgery, Luzhou People's Hospital, Luzhou, Sichuan Province, China
}

\begin{abstract}
Objective: To compare the effect of ursodesoxycholic acid (UDCA) with S-adenosyl-L-methionine (SAMe) plus UDCA in handling of cholestasis in neonates, and their influences on serum endotoxin, matrix metalloproteinase-9 (MMP-9) and interleukin 18 (IL-18).

Study Design: Randomised controlled trial.

Place and Duration of Study: Luzhou People's Hospital, China, from April 2019 to December 2020.

Methodology: A total of 102 neonates with cholestasis were evenly divided into control group and observation group with random double-blind. The former treated with UDCA tablets, and the latter was treated with SAMe plus UDCA. Serum endotoxin, MMP-9 and IL-18 were compared between the two groups.

Results: Total effective rate of treatment in observation group was $92.16 \%$ (47 cases), which was higher than that in control group $70.59 \%$ (36 cases) ( $p=0.005$ ). Levels of serum endotoxin, MMP-9 and IL-18 in observation group were respectively $11.12 \pm$ $1.07 \mathrm{pg} / \mathrm{mL}, 646.72 \pm 42.56 \mathrm{ng} / \mathrm{mL}$ and $2.51 \pm 0.19 \mathrm{pg} / \mathrm{mL}$, which was lower than those in control group $(p<0.001, p=0.007$ and $\mathrm{p}<0.001$, respectively) after 10 days of treatment.

Conclusion: Compared with UDCA alone, SAMe plus UDCA can more effectively improve the curative effect of neonatal cholestasis, and reduce serum endotoxin, MMP-9 and IL-18 levels.
\end{abstract}

Key Words: Ursodesoxycholic acid (UDCA), Neonates, Cholestasis, Endotoxin, Matrix metalloproteinase-9 (MMP-9), Interleukin 18 (IL-18).

How to cite this article: Zeng L, Li M, Cen Y, Wang Z. Effects of S-adenosyl-L-Methionine Combined with Ursodesoxycholic Acid on Serum Endotoxin, MMP-9 and IL-18 in Neonates with Cholestasis. J Coll Physicians Surg Pak 2021; 31(12):1445-1448.

\section{INTRODUCTION}

Cholestasis is the most common clinical manifestation of neonatal liver disease. ${ }^{1}$ Stool of neonates with cholestasis is gray-white due to intestinal bile acid deficiency. Intestinal bile acid reduction can also cause fat absorption disorder, resulting in slow or even stagnant growth rate in these neonates. At present, there is no specific drug for the treatment of neonatal cholestasis. ${ }^{2}$

S-adenosyl-L-methionine (SAMe) is a commonly used drug to protect liver and reduce jaundice. ${ }^{3}$ It can promote the transformation of bile acid through sulfation, improve the detoxification function of bile acid metabolism system, and alleviate the cell damage of oxygen radical, induced by bileacid.

Correspondence to: Zuanyu Wang, Department of Hepatobiliary Pancreatic and Splenic Surgery, Luzhou People's Hospital, Luzhou, Sichuan Province, China

E-mail:d1keh1@163.com

Received: June 21, 2021; Revised: August 21, 2021;

Accepted: October 23, 2021

DOI: https://doi.org/10.29271/jcpsp.2021.12.1445
Previous research has found that SAMe can reduce the incidence of parenteral nutrition-associated cholestasis (PNAC) in very low birth weight infants. ${ }^{4}$

Ursodesoxycholic acid (UDCA) is an endogenous cholic acid, which can promote the secretion of bile acid and induce the change of bile acid composition, increasing the content of UDCA in bile, so as to play a role in choleresis. ${ }^{5}$ UDCA can significantly reduce the concentration of cholesterol and cholesterol ester in human bile, increase the activity of catalase in liver, and enhance the detoxification and antitoxic ability of liver. ${ }^{6}$ One study reported that UDCA could improve the course of very low birth weight infants with PNAC.

Endotoxins may be involved in the occurrence of PNAC. ${ }^{8}$ Endotoxin can promote hepatocyte apoptosis in cholestasis. ${ }^{9}$ Reducing serum endotoxin level can improve clinical condition in premature infants with PNAC. ${ }^{10}$ Studies have confirmed that serum matrix metalloproteinase-9 (MMP-9) can beused in diagnosis of intrahepatic cholestasis of pregnancy (ICP). ${ }^{11}$ Serum interleukin-18 (IL-18) level was significantly higher in patients with severe ICP than in patients with mild ICP. ${ }^{12} \mathrm{~A}$ research found that IL-18 was a valuable biomarkers in diagnosis of 
ICP. ${ }^{13}$ At present, there are no reports about the effects of SAMe combined with UDCA on serum endotoxin, MMP-9 and IL-18 in neonatalcholestasis.

The objective of this study was to compare the effect of UDCA with SAMe plus UDCA in handling of cholestasis in neonates, and their influences on serum endotoxin, MMP-9 and IL-18.

\section{METHODOLOGY}

The randomised controlled trial was conducted at Luzhou People's Hospital, China, from April 2019 to December 2020. Approved by the Ethical Review Committee, a total of 102 neonates with cholestasis were included. Inclusion criteria were that conformed to the diagnostic criteria of neonatal cholestasis; with clinical symptoms such as skin and sclera yellow staining, which was unable to be explained by primary disease; direct bilirubin (DBIL) $>34 \mu \mathrm{mol} / \mathrm{L}$; cholestasis caused by parenteral nutrition, pathological jaundice, $\mathrm{TORCH}$ intrauterine infection, septicemia, etc.; and aged 1-28 days. Exclusion criteria were that neonates with cholestasis caused by congenital defects in bile acid synthesis, hepatitis syndrome, genetic metabolic abnormalities (such as citrin deficiency, progressive familial intrahepatic cholestasis, etc.)

A total of 102 neonates with cholestasis were evenly divided into control group and observation group with random doubleblind, the former treated with UDCA tablets for $10 \mathrm{mg} /(\mathrm{Kg} / \mathrm{d})$ for 10 days; the latter was treated with SAMe plus UDCA for 10 days, namely SAMe was injected intravenously for $50 \mathrm{mg} /$ $(\mathrm{Kg} / \mathrm{d})$, on the basis of UDCA in the control group.

Clinical efficacy was compared. Clinical efficacy was comprehensively judged according to the clinical symptoms and laboratory examination of the neonates after 10 days of treatment. Cured: jaundice dissipated, and total bilirubin, DBIL, alanine aminotransferase, alkaline phosphatase, gamma-glutamyl transferase, total bile acid returned to normal. Improved: jaundice was alleviated, and blood biochemical index significantly decreased than before treatment. Ineffective: symptoms, signs and biochemical indexes did not change or even were aggravated. ${ }^{14}$

Total effective rate $=$ cured rate + improved rate .

Levels of serum endotoxin, MMP-9 and IL-18 were compared before and 10 days after treatment. Adverse events such as tachycardia, liver damage, pruritus, diarrhea and rash were recorded.

SPSS version 25 software was used to analyse the data. Normal distribution test was done with Kolmogorov-Smirnov. Measurement data conforming to normal distribution with Kolmogorov-Smirnov wereexpressed by mean \pm SD, and Independentsamplet-testwasused. Count data such as total effective rate, total adverse events and so on were expressed by $n$ $(\%)$, and Chi-square test was used. The $p<0.05$ indicates that the difference is statistically significant.

\section{RESULTS}

A total of 60 males (58.82\%) and 42 females (41.18\%) were included, with an average age of $17.07 \pm 5.59$ days. The body weight was $2-4 \mathrm{~kg}$, with a mean of $3.06 \pm 0.26 \mathrm{~kg}$.

Total effective rate of treatment in observation group was $92.16 \%$ (47 cases), which was higher than that in control group $70.59 \%$ ( 36 cases) $(p=0.005$, Tablel).

Before treatment, serum endotoxin, MMP-9 and IL-18 levels between the two groups were of no difference $(p=0.717,0.689$ and 0.339 , respectively, Table II). However, after 10 days of treatment, serum endotoxin, MMP-9 and IL-18 in observation group were $11.12 \pm 1.07 \mathrm{pg} / \mathrm{mL}, 646.72 \pm 42.56 \mathrm{ng} / \mathrm{mL}$ and 2.51 $\pm 0.19 \mathrm{pg} / \mathrm{mL}$, respectively, which were lower than those in control group ( $p<0.001, p=0.007$ and $p<0.001$, respectively, Tablell).

In control group, the total adverse events were eight cases (15.69\%), namely, tachycardia one case (1.96\%), liver damage two cases $(3.92 \%)$, pruritus one case $(1.96 \%)$, diarrhea two cases $(3.92 \%)$, and rash two cases (3.92\%). In observation group, the total adverse events were six cases (11.76\%), namely, tachycardia one case (1.96\%), liver damage (1.96\%), pruritus $(1.96 \%)$, and diarrhea in one case $(1.96 \%)$ each, and rash in two cases $(3.92 \%)$. Incidence of adverse events was no difference in control group versus observation group $(p=0.565)$.

Table I: Comparison of curative effects between the two groups.

\begin{tabular}{|l|c|c|c|}
\hline Parameter & $\begin{array}{c}\text { Control group } \\
(\mathbf{n = 5 1 )}\end{array}$ & $\begin{array}{c}\text { Observation } \\
\text { group } \\
(\mathbf{n = 5 1 )}\end{array}$ & p-value \\
\hline Cured [n (\%)] & $20(39.22)$ & $29(56.86)$ & - \\
\hline Improved [n (\%)] & $16(31.37)$ & $18(35.29)$ & - \\
\hline Ineffective [n (\%)] & $15(29.41)$ & $4(7.84)$ & - \\
\hline Total effective rate [n (\%)] & $36(70.59)$ & $47(92.16)$ & 0.005 \\
\hline
\end{tabular}

Table II: Comparison of serum biochemical indexes between the two groups.

\begin{tabular}{|l|c|c|c|}
\hline Parameter & $\begin{array}{c}\text { Control group } \\
(\mathbf{n = 5 1 )}\end{array}$ & $\begin{array}{c}\text { Observation } \\
\text { group } \\
(\mathbf{n}=\mathbf{5 1})\end{array}$ & p-value \\
\hline $\begin{array}{l}\text { Serum endotoxin before treatment } \\
(\mathrm{pg} / \mathrm{mL})\end{array}$ & $27.66 \pm 3.23$ & $27.89 \pm 3.06$ & 0.717 \\
\hline $\begin{array}{l}\text { Serum endotoxin after 10 days of } \\
\text { treatment }(\mathrm{pg} / \mathrm{mL})\end{array}$ & $12.41 \pm 1.13$ & $11.12 \pm 1.07$ & $<0.001$ \\
\hline $\begin{array}{l}\text { Serum MMP-9 before treatment } \\
(\mathrm{ng} / \mathrm{mL})\end{array}$ & $\begin{array}{c}684.65 \pm \\
46.14\end{array}$ & $\begin{array}{c}688.63 \pm \\
53.79\end{array}$ & 0.689 \\
\hline $\begin{array}{l}\text { Serum MMP-9 after 10 days of } \\
\text { treatment }(\mathrm{ng} / \mathrm{mL})\end{array}$ & $\begin{array}{c}658.67 \pm \\
38.03\end{array}$ & $\begin{array}{c}646.72 \pm \\
42.56\end{array}$ & 0.007 \\
\hline $\begin{array}{l}\text { Serum IL-18 before treatment } \\
\text { (pg/mL) }\end{array}$ & $4.11 \pm 0.33$ & $4.17 \pm 0.32$ & 0.339 \\
\hline $\begin{array}{l}\text { Serum IL-18 after 10 days of } \\
\text { treatment }(\mathrm{pg} / \mathrm{mL})\end{array}$ & $2.92 \pm 0.46$ & $2.51 \pm 0.19$ & $<0.001$ \\
\hline
\end{tabular}

\section{DISCUSSION}

The etiology of neonatal cholestasis is complex, and it mainly includes diseases involving in intrahepatic and extrahepatic bile ducts and/or hepatocytes. ${ }^{15}$ Some studies have shown that the endotoxin released during bacterial infection can affect the activity of organic anion transporter on the basement membrane 
of hepatocytes and the activity of RNA processing enzyme 2 in capillary bile duct, resulting in the intake, processing and excretion disorder of bilirubin by hepatocytes, thus inducing cholestasis. ${ }^{16}$

Endotoxin participates in occurrence of cholestasis. ${ }^{17}$ This study showed that comparing with control group, serum endotoxin in observation group was lower, after 10 days of treatment, suggesting that SAMe plus UDCA can reduce the level of serum endotoxin in neonates compared with UDCA alone.

It has been reported that MMP-9 is associated with ICP, inhibition of MMP-9 level can improve ICP symptoms. ${ }^{18}$ This study showed that comparing with control group, serum MMP-9 level in observation group was lower, after 10 days of treatment, suggesting that SAMe plus UDCA can reduce the level of serum MMP-9 in neonates compared with UDCA alone.

The increased expression of IL-18 can induce Th1 type immune response, aggravate the injury of hepatocytes, and promote the secretion of Th1 cytokines by monocytes and phagocytes, thus promoting the progress of the disease ${ }^{19,20}$ This study showed that, comparing with control group, serum IL-18 level in observation group was lower, after 10 days of treatment, suggesting that SAMe plus UDCA can reduce serum IL-18 in neonates compared with UDCA alone.

Some studies pointed out that UDCA could be used for treatment in PNAC in neonates. ${ }^{21,22}$ This study showed that SAMe plus UDCA was more effective than UDCA alone in treatment of neonatal cholestasis. The results of this study are basically consistent with those of previous study. ${ }^{14}$ The reason may be that SAMe combined with UDCA can more effectively reduce serum endotoxin, MMP-9 and IL-18 levels in neonatal cholestasis compared with UDCA alone, and thus the curative effect is better.

\section{CONCLUSION}

Compared with UDCA alone, SAMe plus UDCA can more effectively improve the curative effect of neonatal cholestasis, and reduce serum endotoxin, MMP-9 and IL-18 levels.

\section{ETHICALAPPROVAL:}

This study was approved by the Ethical Review Committee of the Luzhou People's Hospital, Sichuan Province, China.

\section{CONFLICT OF INTEREST:}

The authors declared no conflict of interest.

\section{AUTHORS' CONTRIBUTION:}

LZ: Writing, result interpretation and discussion.

ML, YC: Data collection and analysis, study design

ZW: Conception, revision and final approval.

\section{REFERENCES}

1. Bhatia V, Bavdekar A, Matthai J, Waikar Y, Sibal A. Management of neonatal cholestasis: consensus statement of the pediatric gastroenterology chapter of Indian academy of pediatrics. Indian Pediatr 2014;
51(3):203-11. doi: 10.1007/s13312-014-0375-2.

2. Feldman AG, Sokol RJ. Recent developments in diagnostics and treatment of neonatal cholestasis. Semin Pediatr Surg 2020; 29(4):150945-56. doi:10.1016/ j.sempedsurg.2020.150945.

3. Du XF, Chen J, Ren S, Zhang XD, Huang CY, Shan J. Effect of S-adenosyl-L-methionine in patients with primary biliary cholangitis treated with ursodeoxycholic acid. Chin Hepatol 2021; 26(6):661-3.

4. Li SF, Zhao SY, Zhang H, Ma XH. Preventive effect of Sadenosyl-L-methionine on PNAC in very low birth weigh infants. China Prac Med 2012; 7(15):30-1.

5. Namisaki T, Fujinaga Y, Moriya K, Yoshiji H. The association of histological progression with biochemical response to ursodeoxycholic acid in primary biliary cholangitis. Hepatol Res 2021; 51(1): 31-8. doi:10.1111/ hepr.13593.

6. Kim DJ, Yoon S, Ji SC, Yang J, Kim Y, Lee S, et al. Ursodeoxycholic acid improves liver function via phenylalanine/ tyrosine pathway and microbiome remodelling in patients with liver dysfunction. Sci Rep 2018; 8(1):11874-85. doi:10.1038/s41598-018-30349-1.

7. Chen CY, Tsao PN, Chen HL, Chou HC, Hsieh WS, Chang MH. Ursodeoxycholic acid (UDCA) therapy in very-lowbirth-weight infants with parenteral nutrition-associated cholestasis. J Pediatr 2004; 145(3):317-21. doi:10.1016/j. jpeds.2004.05.038

8. Teitelbaum DH, Tracy T. Parenteral nutrition-associated cholestasis. Semin Pediatr Surg 2001; 10(2):72-80. doi:10.1053/spsu.2001.22386.

9. Moazzam FN, Brems JJ, Yong SL, Filkins JP, Fisher SG, Holt $\mathrm{DR}$, et al. Endotoxin potentiates hepatocyte apoptosis in cholestasis. J Am Coll Surg 2002; 194(6):731-9. doi: 10.1016/s1072-7515(02)01173-0.

10. Liu YF, Zou CC. Effects of ursodeoxycholic acid on premature infants with parenteral nutrition associated cholestasis and on serum endotoxin level. Chin Hosp Pharm J 2017; 37(5):459-61.

11. Chen Z, Shen Z, Hu L, Lu M, Feng Y. Identification of matrix metalloproteinase- 2 and 9 as biomarker of intrahepatic cholestasis of pregnancy. Ann Hepatol 2017; 16(2):291-6. doi: 10.5604/16652681.1231589.

12. Shao Y, Chen J, Zheng J, Liu CR. Effect of histone deacetylase HDAC3 on cytokines IL-18, IL-12 and TNF- $\alpha$ in patients with intrahepatic cholestasis of pregnancy. Cell Physiol Biochem 2017; 42(4):1294-302. doi: 10.1159/000 478958.

13. Abdel-Aziz YR, Mahmoud MS. Biomarkers for diagnosis of intrahepatic cholestasis of pregnancy. Int J Life Sci 2017; 7(1):48-52.

14. Yin C. Clinical observation of ademetionine1, 4butanedisulfonate combined with ursodeoxycholic acid in the treatment of neonatal parenteral nutrition associated cholestasis. China Prac Med 2020; 15(19):99-101. doi:10.14163/j.cnki.11-5547/r.2020.19.043.

15. Silva ES, Silva HM, Catarino C, Dias CC, Santos-Silva A, 
Lopes A. Neonatal cholestasis: development of a diagnostic decision algorithm from multivariate predictive models. Eur J Pediatr 2021; 180(5):1477-86. doi: 10. 1007/s00431-020-03886-Z.

16. Hilscher MB, Kamath PS, Eaton JE. Cholestatic liver diseases: A primer for generalists and subspecialists. Them Rev Gastroent Dis 2020; 95(10):2263-79. doi:10.1016/j. mayocp.2020.01.015.

17. Trauner $M$, Fickert $P$, Stauber RE. Inflammation-induced cholestasis. J Gastroen Hepatol 1999; 14(10):946-59. doi:10.1046/j.1440-1746.1999.01982.x.

18. Zhang M, Xu M. Epigallocatechin-3-gallate ameliorates intrahepatic cholestasis of pregnancy by inhibiting matrix metalloproteinase-2 and matrix metalloproteinase-9. Fund Clin Pharmacol 2017; 31(5):526-33. doi:10.1111/ fcp.12294.
19. Yasuda K, Nakanishi K, Tsutsui H. Interleukin-18 in health and disease. Int J Mol Sci 2019; 20(3):649-703. doi:10.3390/ijms20030649.

20. Zhang L, Zhou X, Dai Y, Lv C, Wu J, Wu Q, et al. Establishment of interleukin-18 time-resolved fluorescence immunoassay and its preliminary application in liver disease. J Clin Lab Anal 2021; 35(5):e23758-65. doi:10. 1002/jcla.23758.

21. Lewis T, Kuye S, Sherman A. Ursodeoxycholic acid versus phenobarbital for cholestasis in the neonatal intensive care unit. BMC Pediatr 2018; 18(1):197-202. doi:10.1186/s12887-018-1167-y.

22. Al-Hathlol K, Al-Madani A, Al-Saif S, Abulaimoun B, AlTawil K, El-Demerdash A. Ursodeoxycholic acid therapy for intractable total parenteral nutrition-associated cholestasis in surgical very low birth weight infants. Singap Med J 2006; 47(2):147-51. 